Research Article

\title{
Antiobesity and Antidiabetic Effects of Portulaca oleracea Powder Intake in High-Fat Diet-Induced Obese C57BL/6 Mice
}

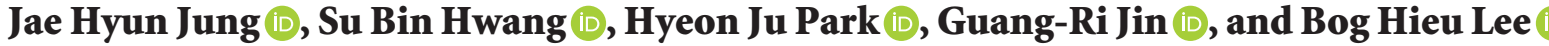 \\ Department of Food and Nutrition, Chung-Ang University, Gyeonggi-do 17546, Republic of Korea \\ Correspondence should be addressed to Bog Hieu Lee; lbheelb@cau.ac.kr
}

Received 25 January 2021; Accepted 30 April 2021; Published 28 June 2021

Academic Editor: Rômulo Dias Novaes

Copyright ( 2021 Jae Hyun Jung et al. This is an open access article distributed under the Creative Commons Attribution License, which permits unrestricted use, distribution, and reproduction in any medium, provided the original work is properly cited.

\begin{abstract}
This study investigated the hypothesis that Portulaca oleracea L. exerts antiobesity and antidiabetic effects by evaluating blood lipid profiles, blood glucose control factors, protein expression of lipid metabolism, and insulin sensitivity improvement. Three groups of high-fat diet (HFD) induced obese C57BL/6 mice $(n=8)$ received treatment with low (5\%; HFD + PO5\%) or high (10\%; HFD + PO10\%) concentrations of $P$. oleracea powder for 12 weeks or no treatment (HFD) and were compared with each other and a fourth control group. Weight gain was reduced by $34 \%$ in the HFD + PO10\% group compared to the HFD group. Moreover, the perirenal and epididymal fat contents in the HFD + PO10\% group were 6.3-fold and 1.5-fold, respectively, lower than those in the HFD group. The atherogenic index (AI) and cardiac risk factor (CRF) results in the $P$. oleracea-treated groups were significantly lower than those in the HFD group. The homeostasis model assessment of insulin resistance (HOMA-IR) levels was lower in the HFD + PO10 $\%$ group than in the HFD group. The protein expression levels of the proliferator-activated receptor (PPAR)- $\alpha$, glucose transporter (GLUT) 4 and PPAR- $\gamma$ were upregulated in the HFD + PO10\% group compared to the HFD group. However, the protein expression levels of tumor necrosis factor (TNF)- $\alpha$ were lower in the P. oleracea-treated groups than in the HFD group. Our results demonstrate that $P$. oleracea powder could be effectively used to treat and prevent obesity and diabetes-associated diseases through suppression of weight gain and reduction in body fat and blood glucose levels.
\end{abstract}

\section{Introduction}

The prevalence of chronic diseases such as obesity, type 2 diabetes, and cardiovascular disease has been steadily rising over the past several decades due to the westernization of eating habits, which involve a high intake of high-fat foods and red meat [1]. These changes are increasingly affecting the world population; therefore, the prevalence of dyslipidemia, arteriosclerosis, and cardiovascular disease is growing [2,3]. The prevalence of obesity in Korean males has increased from $25.7 \%$ (phase I) to $37.9 \%$ (phase IV), according to the Korea National Health and Nutrition Examination Survey (KNHANES) [4]. Diabetes is becoming one of the most common health problems in developed countries such as Japan and North America and Europe [5]. In Korea, this disease will affect an estimated 4.3 million people by 2030 [6].

To help control obesity and maintain a normal weight, many studies have focused on appetite control, inhibition of lipid digestion and absorption, acceleration of energy expenditure through adaptive thermogenesis, and modulation of lipid metabolism [7]. Additionally, lipogenesis and lipolysis are important biochemical mechanisms that regulate lipid metabolism [7]. Peroxisome proliferator-activated receptor (PPAR)- $\alpha$, which is associated with lipolysis, is abundantly expressed in brown fat and in the liver. These receptors mainly inhibit the growth and differentiation of adipocytes and promote lipolysis to regulate lipoprotein synthesis and tissue inflammatory responses. PPAR- $\alpha$ is also known to stimulate carnitine palmitoyltransferase (CPT)-1, which facilitates the transport of free fatty acids (FFAs) to mitochondria, thereby promoting $\beta$-oxidation of FFAs [8].

Adiponectin, the expression of which is regulated by factors such as PPAR- $\gamma$, reduces body weight and blood glucose while enhancing insulin sensitivity [9]. PPAR- $\gamma$ is known to be a major regulator of adiponectin gene transcription and an important biological indicator associated 
with the insulin signaling pathway, insulin resistance, and inflammation [9, 10]. In contrast, tumor necrosis factor (TNF) $-\alpha$ has been found to be upregulated in obese patients and those suffering from diabetes and is known to directly contribute to reduced adiponectin expression [10]. Glucose transporter (GLUT) 4 upregulation also ameliorates insulin resistance [11].

Portulaca oleracea $\mathrm{L}$. is an annual plant belonging to the Portulacaceae family and is rich in dopamine, L-noradrenaline, flavonoids, organic acids, coumarin (a type of polyphenol), vitamins (B1 and B2), and $\gamma$-linolenic acid, which is an $\omega-6$ fatty acid [12-14]. P. oleracea has traditionally been used as a remedy for burns, headaches, cough, dyspnea, and arthritis and has been reported to possess several therapeutic properties such as antiulcer, anti-inflammatory, antidiabetic, antioxidant, and antibacterial effects $[15,16]$. Although some studies have been conducted to assess the antiobesity and antidiabetic properties of $P$. oleracea [17-19], few studies have characterized the mechanisms that underlie this the therapeutic effects of this plant. Therefore, our study analyzed the effects of $P$. oleracea on several weight control-associated endpoints including blood glucose reduction and blood lipid profiles. High-fat diet-induced obese C57BL/6 mice $(n=8)$ were fed low (5\%; $\mathrm{HFD}+\mathrm{PO} 5 \%)$ or high $(10 \%$; HFD + PO10\%) concentrations of $P$. oleracea powder $(n=8)$ for 12 weeks, after which the mechanism of $P$. oleracea activity was investigated by characterizing the expression of related genes and proteins.

\section{Materials and Methods}

2.1. Diet Preparation and Formula Composition. The mice obtained for this study were divided into four groups: the control (CON) group, which was fed with normal mouse chow diet, the high-fat diet (HFD) group, the HFD + low (5\%) P. oleracea powder (HFD + PO5\%), and the $\mathrm{HFD}+$ high (10\%) P. oleracea powder (HFD + PO10\%) group. The CON group feed (NIH-41) and HFD group feed (TD.06414) were purchased from Dae Han Bio Link Co. Ltd. (Eumsung, Chungbuk, Korea). Table 1 shows the composition of the experimental diet formula. The composition of P. oleracea powder purchased from Hanbit Farm (Yeongdeok, 73 Kyeongbuk, Korea) was indicated in Table 1.

2.2. Animal Breeding and Experimental Design. Male C57BL/ 6 mice were purchased from Dae Han Bio Link Co. Ltd. (Eumsung, Chungbuk, Korea). Thirty-two 5-week-old male C57BL/6 mice were employed in this study. Feeding environments were maintained at $23 \pm 2^{\circ} \mathrm{C}$, with a relative humidity of $50 \pm 5 \%$, and a $12: 12 \mathrm{~h}$ light-dark cycle. All animal procedures were performed according to the Care and Use of Laboratory Animals guidelines of the National Institutes of Health and the Animal Welfare Act guidelines. The mice were allowed to acclimate to the laboratory environment for one week and provided free access to pelleted food and water. After the acclimation period, the mice were randomly assigned to the four experimental groups ( $n=8$ /group) and raised for 12 weeks. Food intake and body weight were measured weekly and twice per week, respectively. The study was approved by the Institutional Animal Care and Use Committee (IACUC) from Chung-Ang University (approval ID: 2019-00006).

2.3. Blood Glucose Measurement. Blood glucose was measured seven times at $0,2,4,6,8,10$, and 12 weeks after the start of the feeding trials; this procedure was always conducted at the same time of day. Blood was collected from the tail vein, and blood glucose was measured using a blood glucose meter (Accu-Check Performa, Roche Diagnostics, Mannheim, Germany).

2.4. Intraperitoneal Glucose Tolerance Test. After a $12 \mathrm{~h}$ fast, the mice were injected with $10 \mu \mathrm{l} / \mathrm{g}$ body weight (BW) of D-glucose $(100 \mathrm{mg} / \mathrm{ml}$ in saline). Blood was collected from the tail vein at $0,30,60,90$, and $120 \mathrm{~min}$ after injection, and blood glucose was measured using a blood glucose meter (Accu-Check Performa, Roche Diagnostics, Mannheim, Germany).

2.5. Blood and Organ Collection. The mice were euthanized with $\mathrm{CO}_{2}$ gas after fasting for $12 \mathrm{~h}$. Blood was collected via cardiac puncture and allowed to sit at room temperature for $30 \mathrm{~min}$, followed by centrifuging at $3000 \mathrm{rpm}$ for $15 \mathrm{~min}$ at $4^{\circ} \mathrm{C}$. The heart, liver, kidney, spleen, testes, and epididymis with the perirenal fat, epididymal fat, and brown fat were harvested. Afterward, each organ was rinsed with physiological saline, weighed, and stored at $-70^{\circ} \mathrm{C}$ for later use.

2.6. Histopathological Analysis. The liver tissues were fixed with $10 \%$ neutral formalin. Tissue sections were prepared by routine paraffin embedding; $5 \mu \mathrm{m}$-thick tissue slides were stained with hematoxylin and eosin $(\mathrm{H} \& \mathrm{E})$. A veterinary pathologist examined the slides for histopathologic lesions.

2.7. Blood Chemistry Analysis. The levels of serum total cholesterol (TC), triglycerides (TGs), and high-density lipoprotein (HDL)-cholesterol were determined with commercial enzymatic kits coupled with a SPOTCHEMTM EZ SP-4430 automated analyzer (ARKRAY, Inc., Kyoto, Japan). Low-density lipoprotein (LDL)-cholesterol levels were calculated using the Friedewald formula (TCHDL-TG/5). The AI [Atherogenic Index $=(\mathrm{TC}-\mathrm{HDL}-$ cholesterol)/HDL-cholesterol] and CRF (cardiac risk factor $=\mathrm{TC} / \mathrm{HDL}$-cholesterol) were calculated using total serum cholesterol and HDL-cholesterol content [20]. Serum insulin content was analyzed using a mouse insulin ELISA kit (Cusabio Biotech CO., Ltd, USA). Alanine aminotransferase (ALT) levels were also analyzed using an enzymatic colorimetric test. The homeostasis model assessment of insulin resistance (HOMA-IR) was calculated based on fasting insulin levels and fasting glucose levels [21]. HOMA-IR was calculated using the following formula: 
TABLE 1: Composition of diet formula (\%).

\begin{tabular}{|c|c|c|c|c|c|}
\hline Ingredient & $\mathrm{CON}^{(1)}$ & Ingredient & $\mathrm{HFD}^{(2)}$ & HFD + PO5 $\%$ & $\mathrm{HFD}+\mathrm{PO} 10 \%$ \\
\hline Ground whole hard meal & 34.90 & Casein & 26.50 & 25.18 & 23.85 \\
\hline Ground ${ }^{\#} 2$ yellow corn & 21.00 & L-cysteine & 0.40 & 0.38 & 0.36 \\
\hline Ground whole oats & 10.00 & Maltodextrin & 16.00 & 15.2 & 14.4 \\
\hline Wheat middlings & 10.00 & Sucrose & 9.00 & 8.55 & 8.10 \\
\hline Fish meal ( $60 \%$ protein $)$ & 9.00 & Lard & 31.00 & 29.45 & 27.9 \\
\hline Soy oil & 2.00 & Soybean oil & 3.00 & 2.85 & 2.7 \\
\hline Soybean meal ( $47.5 \%$ protein) & 5.00 & Cellulose & 6.55 & 6.55 & 5.90 \\
\hline Alfalfa meal (17\% protein) & 2.00 & Mineral mix ${ }^{(3)}$ & 4.80 & 4.56 & 4.32 \\
\hline Corn gluten meal ( $60 \%$ protein) & 2.00 & Calcium phosphate & 0.34 & 0.32 & 0.31 \\
\hline Dicalcium phosphate & 1.50 & Vitamin $\operatorname{mix}^{(4)}$ & 2.10 & 2.0 & 1.89 \\
\hline Yeast-brewer & 1.00 & Choline bitartrate & 0.30 & 0.29 & 0.27 \\
\hline Premixes & 0.60 & Blue food color & 0.01 & 0.01 & 0.01 \\
\hline Ground limestone & 0.50 & Portulaca oleracea powder ${ }^{(5)}$ & - & 5.00 & 10.00 \\
\hline Salt & 0.50 & Energy $(\mathrm{kcal} / \mathrm{g})^{(6)}$ & 5.10 & 4.86 & 4.61 \\
\hline Energy (kcal/g) & 3.11 & - & & & \\
\hline
\end{tabular}

${ }^{(1)} \mathrm{CON}$ : control diet; HFD: high fat diet; HFD + PO5\%: high fat diet + 5\% P. oleracea powder; HFD + PO10\%: high fat diet + $10 \%$ P. oleracea powder. ${ }^{(2)} \mathrm{HFD}$ was composed of $60 \% \mathrm{kcal}$ from fat (protein $23.5 \%$, carbohydrate $27.3 \%$, and fat $34.3 \%$ by weight). ${ }^{(3)}$ Mineral mixture according to AIN-93G-MX. ${ }^{(4)}$ Vitamin mixture according to AIN-93-VX. ${ }^{(5)}$ The powder was purchased from Hanbit Farm (Yeongdeok, Kyeongbuk, Korea) and composed of protein 13.4\%, fat $1.9 \%$, nitrogen-free extract $47.6 \%$, ash $12.1 \%$, and fiber $25.0 \%$ based on Standard Tables of Feed Composition from Korea National Institute of Animal Science.

${ }^{(6)}$ Calculated by Portulaca oleracea nutrient based on Food Data Central of U.S Department of Agriculture.

2.8. Western Blot. Liver tissue $(80 \mathrm{mg})$ was homogenized in RIPA buffer $(800 \mu \mathrm{l})$ (Thermo Scientific, Rockford, IL). After centrifugation at $14,000 \mathrm{rpm}$ for $15 \mathrm{~min}$ at $7^{\circ} \mathrm{C}$, the supernatant was used to quantify PPAR- $\alpha$, PPAR- $\gamma$, TNF- $\alpha$, and GLUT4 protein expression levels via western blot analysis. The total protein content of the supernatant was determined with the BCA reagent method (Thermo scientific, Bartlesville, USA). Equal amounts of protein $(30 \mu \mathrm{g})$ were separated by $10 \%$ sodium dodecyl sulfate polyacrylamide gel electrophoresis and transferred to a nitrocellulose membrane (Hybond, GE Healthcare Life Science, Little Chalfont, UK). The membrane was blocked for $2 \mathrm{~h}$ at room temperature with $5 \%$ skimmed milk. After overnight incubation at $4^{\circ} \mathrm{C}$ with either primary anti-mouse PPAR- $\alpha$ (Santa Cruz. USA), anti-rabbit PPAR- $\gamma$ (Cell Signaling Technology, Inc.), antirabbit TNF- $\alpha$ (Cell Signaling Technology, Inc.), or glucose transporter type 4 (GLUT4) (Cell Signaling Technology, Inc.) antibodies, the membrane was washed with TBST and incubated with a horseradish peroxidase-conjugated secondary goat anti-rabbit IgG antibody and a horse antimouse IgG (Cell Signaling Technology, Inc.) antibody for $1.5 \mathrm{~h}$ at room temperature. Immunodetection was carried out using an ECL detection reagent (SuperSignal, Thermo Scientific, Rockford, IL., USA). All figures showing the results of quantitative analyses (ImageJ, National Institute of Health) include data from at least three independent experiments.

2.9. Statistical Analysis. SPSS version 25 (IBM Corp., Armonk, NY, USA) was used for statistical analysis, and all experimental data are presented as the mean \pm standard error (SE). One-way ANOVA was performed to test whether the means were significantly different among the groups. Duncan's multiple range analysis was used as a post hoc test to analyze the differences between group pairs. A $p$ value $<0.05$ was considered statistically significant.

\section{Results}

3.1. Final Body Weight, Weight Gain, Food Intake, and Food Efficiency Ratio. The final body weight, weight gain, food intake, and food efficiency ratio (FER) are summarized in Table 2. The final body weight was higher in the HFD groups than in the CON group $(p<0.05)$, whereas, in the HFD groups, the final body weight of the HFD $+\mathrm{PO} 10 \%$ group was $13 \%$ lower than that in the HFD group $(p<0.05)$. There was no significant difference between the HFD group and the HFD + PO5\% group. Weight gain was significantly higher in the HFD groups than in the CON group. When comparing weight gains exclusively between the HFD groups, the weight gain in the HFD + PO10\% group was $34 \%$ lower than that in the HFD group $(p<0.05)$. Food intake was higher in the CON, HFD + PO5\%, and HFD + PO10\% groups than in the HFD group $(p<0.05)$, with no significant differences between the three groups. The FER was significantly higher in the HFD groups than in the CON group; however, the FER of the HFD + PO10\% group was lower than those of both the HFD and HFD + PO5\% groups. Energy intake was higher in the HFD groups than in the CON group $(p<0.05)$, but there were no significant differences among the HFD groups.

3.2. Organ Weight and Unit Organ Weight. Table 3 shows the total weight and unit total weight of the major organs and body fat for each group. The liver weight in the HFD group was significantly greater than that in the CON group, whereas that in the HFD + PO10\% group was lower than that in the HFD group $(p<0.05)$. The liver weight in the HFD + PO5\% group was not significantly different from that in the HFD group when comparing only the HFD groups. Furthermore, the heart, kidney, and spleen weights in the HFD groups were significantly greater than those in the CON group, but there were no significant differences among 
TABLE 2: Effect of experimental diets on body weight gain and daily food intake.

\begin{tabular}{|c|c|c|c|c|}
\hline \multirow{2}{*}{ Item } & \multicolumn{4}{|c|}{ Group $^{(1)}$} \\
\hline & $\mathrm{CON}$ & HFD & $\mathrm{HFD}+\mathrm{PO} 5 \%$ & $\mathrm{HFD}+\mathrm{PO} 10 \%$ \\
\hline Initial body weight $(\mathrm{g})$ & $21.7 \pm 0.30^{(3) \mathrm{NS}}$ & $20.7 \pm 0.45$ & $20.9 \pm 0.49$ & $21.8 \pm 0.17$ \\
\hline Final body weight $(\mathrm{g})$ & $27.9 \pm 0.31^{\mathrm{c}}$ & $39.3 \pm 1.67^{\mathrm{a}}$ & $37.8 \pm 1.30^{\mathrm{ab}}$ & $34.2 \pm 1.36^{\mathrm{b}}$ \\
\hline Body weight gain (g/12 weeks) & $6.30 \pm 0.39^{c}$ & $18.6 \pm 1.62^{\mathrm{a}}$ & $16.9 \pm 1.18^{\mathrm{a}}$ & $12.3 \pm 1.32^{\mathrm{b}}$ \\
\hline Daily food intake (g/day) & $2.82 \pm 0.07^{\mathrm{b}}$ & $2.45 \pm 0.07^{\mathrm{a}}$ & $2.62 \pm 0.03^{\mathrm{b}}$ & $2.71 \pm 0.03^{\mathrm{b}}$ \\
\hline $\operatorname{FER}^{(2)}$ & $0.027 \pm 0.002^{c}$ & $0.091 \pm 0.007^{\mathrm{a}}$ & $0.078 \pm 0.006^{\mathrm{a}}$ & $0.054 \pm 0.006^{\mathrm{b}}$ \\
\hline Energy intake (kcal/day) & $8.79 \pm 0.22^{\mathrm{b}}$ & $12.5 \pm 0.34^{\mathrm{a}}$ & $12.7 \pm 0.17^{\mathrm{a}}$ & $12.5 \pm 0.13^{\mathrm{a}}$ \\
\hline
\end{tabular}

${ }^{(1)} \mathrm{CON}$ : control; HFD: high fat diet; HFD + PO5\%: high fat diet + 5\% P. oleracea powder; HFD + PO10\%: high fat diet + $10 \%$ P. oleracea powder. ${ }^{(2)} \mathrm{FER}($ food efficiency ratio) = body weight gain/food intake per week. ${ }^{(3)}$ Values are the mean $\pm \mathrm{SE}\left(n=8 /\right.$ group). ${ }^{\text {(a-c) }}$ Values with a different superscript in the same row are significantly different at $p<0.05$ by Duncan's multiple range test. NS: not significant.

TABLE 3: Organ weight and unit organ weight of C57BL/6 mice fed the experimental diets.

\begin{tabular}{lcccccccc}
\hline \multirow{2}{*}{ Group $^{(1)}$} & \multicolumn{3}{c}{ Total organ weight $(\mathrm{g})$} & \multicolumn{3}{c}{ Total unit organ weight (mg/g BW) } \\
& CON & HFD & HFD + PO5\% & HFD + PO10\% & CON & HFD & HFD + PO5\\
% & HFD + PO10\% \\
\hline Liver & $0.99 \pm 0.012^{(2) \mathrm{b}}$ & $1.08 \pm 0.046^{\mathrm{a}}$ & $1.03 \pm 0.024^{\mathrm{ab}}$ & $0.98 \pm 0.018^{\mathrm{b}}$ & $35.35 \pm 0.331^{\mathrm{a}}$ & $27.43 \pm 0.598^{\mathrm{b}}$ & $27.50 \pm 0.712^{\mathrm{b}}$ & $29.06 \pm 1.124^{\mathrm{b}}$ \\
Heart & $0.10 \pm 0.005^{\mathrm{b}}$ & $0.13 \pm 0.006^{\mathrm{a}}$ & $0.13 \pm 0.006^{\mathrm{a}}$ & $0.12 \pm 0.003^{\mathrm{a}}$ & $3.64 \pm 0.197^{\mathrm{NS}}$ & $3.40 \pm 0.176$ & $3.56 \pm 0.157$ & $3.63 \pm 0.199$ \\
Kidney & $0.33 \pm 0.004^{\mathrm{b}}$ & $0.37 \pm 0.008^{\mathrm{a}}$ & $0.37 \pm 0.009^{\mathrm{a}}$ & $0.36 \pm 0.013^{\mathrm{a}}$ & $11.79 \pm 0.182^{\mathrm{a}}$ & $9.39 \pm 0.363^{\mathrm{c}}$ & $9.91 \pm 0.376^{\mathrm{bc}}$ & $10.70 \pm 0.535^{\mathrm{ab}}$ \\
Spleen & $0.05 \pm 0.003^{\mathrm{b}}$ & $0.06 \pm 0.003^{\mathrm{a}}$ & $0.06 \pm 0.003^{\mathrm{a}}$ & $0.06 \pm 0.002^{\mathrm{a}}$ & $1.61 \pm 0.089^{\mathrm{NS}}$ & $1.61 \pm 0093$ & $1.59 \pm 0.049$ & $1.74 \pm 0.104$ \\
Testis & $0.19 \pm 0.013^{\mathrm{NS}}$ & $0.19 \pm 0.011$ & $0.18 \pm 0.015$ & $0.18 \pm 0.008$ & $6.66 \pm 0.523^{\mathrm{a}}$ & $4.84 \pm 0.413^{\mathrm{b}}$ & $4.82 \pm 0.471^{\mathrm{b}}$ & $5.34 \pm 0.423^{\mathrm{ab}}$ \\
Epididymidis & $0.02 \pm 0.002^{\mathrm{NS}}$ & $0.02 \pm 0.002$ & $0.02 \pm 0.002$ & $0.02 \pm 0.002$ & $0.58 \pm 0.065^{\mathrm{NS}}$ & $0.49 \pm 0.065$ & $0.47 \pm 0.047$ & $0.52 \pm 0.057$ \\
Perirenal fats & $0.51 \pm 0.055^{\mathrm{c}}$ & $2.15 \pm 0.177^{\mathrm{a}}$ & $1.95 \pm 0.093^{\mathrm{a}}$ & $0.34 \pm 0.146^{\mathrm{b}}$ & $18.23 \pm 1.878^{\mathrm{c}}$ & $54.42 \pm 3.006^{\mathrm{a}}$ & $51.79 \pm 2.673^{\mathrm{a}}$ & $38.89 \pm 4.002^{\mathrm{b}}$ \\
Epididymal fats & $0.02 \pm 0.002^{\mathrm{b}}$ & $0.03 \pm 0.003^{\mathrm{a}}$ & $0.03 \pm 0.002^{\mathrm{a}}$ & $0.02 \pm 0.003^{\mathrm{b}}$ & $0.63 \pm 0.061^{\mathrm{NS}}$ & $0.77 \pm 0.070$ & $0.77 \pm 0.065$ & $0.63 \pm 0.089$ \\
tissue & & & & & & & \\
Brown fats & $0.08 \pm 0.004^{\mathrm{bc}}$ & $0.10 \pm 0.011^{\mathrm{ab}}$ & $0.10 \pm 0.008^{\mathrm{a}}$ & $0.07 \pm 0.005^{\mathrm{c}}$ & $2.69 \pm 0.137^{\mathrm{a}}$ & $2.45 \pm 0.240^{\mathrm{ab}}$ & $2.69 \pm 0.209^{\mathrm{a}}$ & $2.04 \pm 0.161^{\mathrm{b}}$ \\
tissue & & & & & & & &
\end{tabular}

${ }^{(1)} \mathrm{CON}$ : control diet; HFD: high fat diet; HFD + PO5\%: high fat diet + 5\% P. oleracea powder; HFD + PO10\%: high fat diet + 10\% P. oleracea powder.

${ }^{(2)}$ Values are the mean \pm SE $\left(n=8 /\right.$ group). ${ }^{(a-c)}$ Values with different superscript in the same row are significantly different at $p<0.05$ by Duncan's multiple range test. NS: not significant.

the HFD groups themselves. No differences were identified between the testicular and epididymal weights among any of the experimental groups.

However, the perirenal fat weight was greater in the HFD and HFD + PO5\% groups than in the CON group, whereas that of the HFD + PO10\% group was significantly less than that of the HFD group (6.3-fold). The epididymal fat weight was significantly less in the CON and $\mathrm{HFD}+\mathrm{PO} 10 \%$ groups than in the HFD and HFD + PO5\% groups (1.5-fold). The brown fat weight was greater in the HFD + PO5\% group than in the CON and $\mathrm{HFD}+\mathrm{PO} 10 \%$ groups but was not different from that in the HFD group.

Regarding the total unit organ weight, although the liver unit organ weight was greater in the CON group than in the HFD groups $(p<0.05)$, there were no significant differences among the HFD groups. The heart and spleen total unit organ weights were also not significantly different among groups. However, the kidney unit organ weight was greater in the CON group than in the HFD and HFD + PO5\% groups $(p<0.05)$ but not significantly different from that in the HFD + PO10\% group. The testicular unit organ weight was significantly greater in the CON group than in the HFD and HFD + PO5\% groups but was not significantly different from that in the HFD + PO10\% group. Additionally, no differences between the epididymal unit organ weights were identified among the groups $(p<0.05)$.

Furthermore, the perirenal fat weight was found to be significantly less in the CON group than in the HFD groups. Upon comparing only the HFD groups, the perirenal fat unit weight in the HFD + PO10\% group was found to be 1.4 -fold less than that in the HFD group; however, there was no significant difference between the weights in the HFD and HFD + PO5\% groups. Regarding the epididymal fat unit weight, the HFD + PO10\% group tended to have lower values than the HFD and the HFD + PO5\% groups; however, the difference between the groups was not significant. There were no differences in the brown fat unit weights in the CON group and the HFD and HFD + PO5\% groups, but that in the $\mathrm{HFD}+\mathrm{PO} 10 \%$ group was less than that in the CON group.

3.3. Blood Chemical Analysis. Table 4 shows the effects of P. oleracea powder on the blood lipid profiles, insulin levels, HOMA-IR values, and liver function tests of obese mice. The TC level was lower in the CON group than in the HFD groups, albeit not significantly. However, the HDL-cholesterol level in the HFD groups was significantly higher than that in the CON group, and the levels in the HFD + PO5\% and HFD + PO10\% groups were higher (1.3- and 1.2-fold, respectively) than that the HFD group $(p<0.05)$. The LDL- 
TABLE 4: Blood serum lipid parameter of C57BL/6 mice fed the experimental diets.

\begin{tabular}{|c|c|c|c|c|}
\hline \multirow{2}{*}{ Item } & \multicolumn{4}{|c|}{ Group $^{(1)}$} \\
\hline & $\mathrm{CON}$ & HFD & $\mathrm{HFD}+\mathrm{PO} 5 \%$ & $\mathrm{HFD}+\mathrm{PO} 10 \%$ \\
\hline $\mathrm{TC}(\mathrm{mg} / \mathrm{dL})$ & $59.2 \pm 3.4^{(6) b}$ & $81.8 \pm 6.4^{\mathrm{a}}$ & $89.5 \pm 5.5^{\mathrm{a}}$ & $92.6 \pm 0.5^{\mathrm{a}}$ \\
\hline HDL-cholesterol (mg/dL) & $44.3 \pm 3.0^{c}$ & $58.3 \pm 5.8^{\mathrm{b}}$ & $75.0 \pm 4.9^{\mathrm{a}}$ & $72.5 \pm 0.2^{\mathrm{a}}$ \\
\hline LDL-cholesterol (mg/dL) & $6.3 \pm 0.3^{c}$ & $11.7 \pm 0.4^{\mathrm{a}}$ & $11.3 \pm 0.8^{\mathrm{ab}}$ & $9.8 \pm 0.7^{\mathrm{b}}$ \\
\hline Triglyceride $(\mathrm{mg} / \mathrm{dL})$ & $43.5 \pm 1.0^{\mathrm{c}}$ & $59.5 \pm 1.4^{\mathrm{a}}$ & $46.0 \pm 3.7^{\mathrm{bc}}$ & $51.5 \pm 1.9^{\mathrm{b}}$ \\
\hline TG/HDL-cholesterol & $1.01 \pm 0.05^{\mathrm{a}}$ & $1.08 \pm 0.08^{\mathrm{a}}$ & $0.64 \pm 0.07^{\mathrm{b}}$ & $0.71 \pm 0.03^{\mathrm{b}}$ \\
\hline $\mathrm{AI}^{(2)}$ & $0.34 \pm 0.01^{\mathrm{b}}$ & $0.43 \pm 0.03^{\mathrm{a}}$ & $0.29 \pm 0.03^{\mathrm{b}}$ & $0.28 \pm 0.01^{\mathrm{b}}$ \\
\hline $\mathrm{CRF}^{(3)}$ & $1.34 \pm 0.01^{\mathrm{b}}$ & $1.43 \pm 0.03^{\mathrm{a}}$ & $1.29 \pm 0.03^{\mathrm{b}}$ & $1.28 \pm 0.01^{\mathrm{b}}$ \\
\hline Insulin $(\mu \mathrm{U} / \mathrm{ml})$ & $18.8 \pm 2.85^{\mathrm{b}}$ & $39.3 \pm 4.92^{\mathrm{a}}$ & $39.4 \pm 2.68^{\mathrm{a}}$ & $27.5 \pm 5.23^{\mathrm{ab}}$ \\
\hline $\mathrm{HOMA}^{-\mathrm{IR}^{(4)}}$ & $4.2 \pm 0.7^{\mathrm{b}}$ & $10.6 \pm 2.2^{\mathrm{a}}$ & $7.6 \pm 1.4^{\mathrm{ab}}$ & $5.2 \pm 1.5^{\mathrm{b}}$ \\
\hline $\operatorname{ALT}(\mathrm{U} / \mathrm{L})^{(5)}$ & $53.0 \pm 8.7^{\mathrm{b}}$ & $135.5 \pm 14.0^{\mathrm{a}}$ & $46.8 \pm 3.5^{\mathrm{b}}$ & $40.3 \pm 4.5^{\mathrm{b}}$ \\
\hline
\end{tabular}

${ }^{(1)} \mathrm{CON}$ : control diet; HFD: high fat diet; HFD + PO5\%: high fat diet $+5 \%$ P. oleracea powder; HFD + PO10\%: high fat diet + 10\% P. oleracea powder. ${ }^{(2)} \mathrm{AI}$ (atherogenic index) $=$ total cholesterol-HDL-cholesterol/HDL-cholesterol. ${ }^{(3)} \mathrm{CRF}$ (cardiac risk factor) $=$ total cholesterol/HDL cholesterol. ${ }^{(4)} \mathrm{HOMA}-\mathrm{IR}$ (homeostasis model assessment of insulin resistance) = fasting insulin level $(\mu \mathrm{U} / \mathrm{mL})^{*}$ fasting glucose level $(\mathrm{mg} / \mathrm{dL}) / 405 .{ }^{(5)}$ Alanine aminotransferase ${ }^{(6)}$ Values are the mean \pm SE ( $n=8$ /group). ${ }^{(a-c)}$ Values with a different superscript in the same row are significantly different at $p<0.05$ by Duncan's multiple range test.

cholesterol level was lower in the CON group than in the HFD groups, and the level in the HFD + PO10\% group was $16.2 \%$ lower than that in the HFD group; however, this value was not significantly different from that in the HFD + PO5\% group. The TG level was lower in the CON group than in the HFD and HFD + PO10\% groups, but there was no significant difference between the HFD group and the HFD + PO5\% group. In contrast, the TG levels in the HFD + PO5\% and HFD + PO10\% groups were lower than those in the HFD group $(p<0.05)$. The TG/HDL-cholesterol index was significantly lower in the HFD + PO5\% and HFD + PO10\% groups than in the CON and HFD groups. Additionally, the CON, HFD + PO5\%, and HFD + PO10\% groups exhibited lower AI and CRF values than those in the HFD group, but no significant differences were identified among the three aforementioned groups.

The serum insulin level was significantly higher in the HFD and $\mathrm{HFD}+\mathrm{PO} 5 \%$ groups than in the CON group, whereas there were no differences between the $\mathrm{CON}$ and HFD + PO10\% groups. Likewise, the HOMA-IR value, an insulin resistance index, was higher in the HFD and $\mathrm{HFD}+\mathrm{PO} 5 \%$ groups than in the CON group. On the other hand, the HOMA-IR value in the HFD + PO10\% group was lower than that in the HFD group $(p<0.05)$, but there were no differences between the CON and $\mathrm{HFD}+\mathrm{PO} 10 \%$ groups. ALT, a liver injury index, was the highest in the HFD group $(p<0.05)$, with no significant differences among the remaining three groups (CON, HFD + PO5\%, and HFD + PO10\%).

3.4. Random Blood Glucose Measurement. Figure 1 shows the random blood glucose measurements for experimental weeks $0,2,4,6,8,10$, and 12 (all measurements were obtained at the same time of day). There were no significant differences among groups on weeks $0,2,4,6$, and 8 . However, the random blood glucose level in week 10 was lower in the CON group than in the HFD groups. When comparing only the three HFD groups, the HFD + PO5\% and the HFD + PO10\% groups exhibited lower blood glucose levels than the HFD group. The random blood glucose levels in week 12 were found to be similar to those in week 10 .
However, not only was the random blood glucose level found to be lower in the CON, $\mathrm{HFD}+\mathrm{PO} 5 \%$, and HFD + PO10\% groups than in the HFD group, but lower glucose levels were also recorded compared to week 10.

3.5. Intraperitoneal Glucose Tolerance Test. Figure 2 shows the results of the intraperitoneal glucose tolerance test (IPGTT). At the beginning of each experiment, the blood glucose level in each group ranged from $88-129 \mathrm{mg} / \mathrm{dl}$ at minute 0. After $30 \mathrm{~min}$, blood glucose levels increased rapidly by a factor of 2.6 to 3.6 compared to the initial blood glucose concentrations and also tended to decrease from 60 to $120 \mathrm{~min}$. After $120 \mathrm{~min}$, when the experiment was concluded, the blood glucose level in the HFD + PO10\% group $(138.5 \mathrm{mg} / \mathrm{dL})$ was found to be the lowest $(p<0.05)$; however, the difference between the value in this group and that in the CON group $(150.9 \mathrm{mg} / \mathrm{dL})$ was not deemed significant. The value in the HFD group was found to be the highest among the experimental groups. When comparing the changes in blood glucose levels at $120 \mathrm{~min}$ versus $30 \mathrm{~min}$ for each experimental group, the level in the HFD group decreased by $39 \%$, and the levels in the CON group, HFD + PO5\% group, and HFD + PO10 group decreased by $53 \%, 43 \%$, and $51 \%$, respectively.

3.6. Effect of P. oleracea Powder on Liver Morphology. The effects of $P$. oleracea powder on lipid accumulation and cellular swelling in the liver were determined by H\&E staining. Considerable lipid accumulation was observed in the livers of HFD-fed mice, as determined by H\&E staining (Figure $3(\mathrm{~b})$ ). In contrast, $P$. oleracea powder alleviated lipid accumulation to the extent that the HFD + PO10\% group showed similar staining to that of the CON group (Figures 3(a), 3(c), and 3(d)). Furthermore, morphological analyses indicated that the lipid droplet size was the largest in the HFD group, followed by the HFD + PO5\% group, the HFD + PO10\% group, and the CON group (Figure 3). Histopathological lesions of the liver were not observed in the CON group. However, 


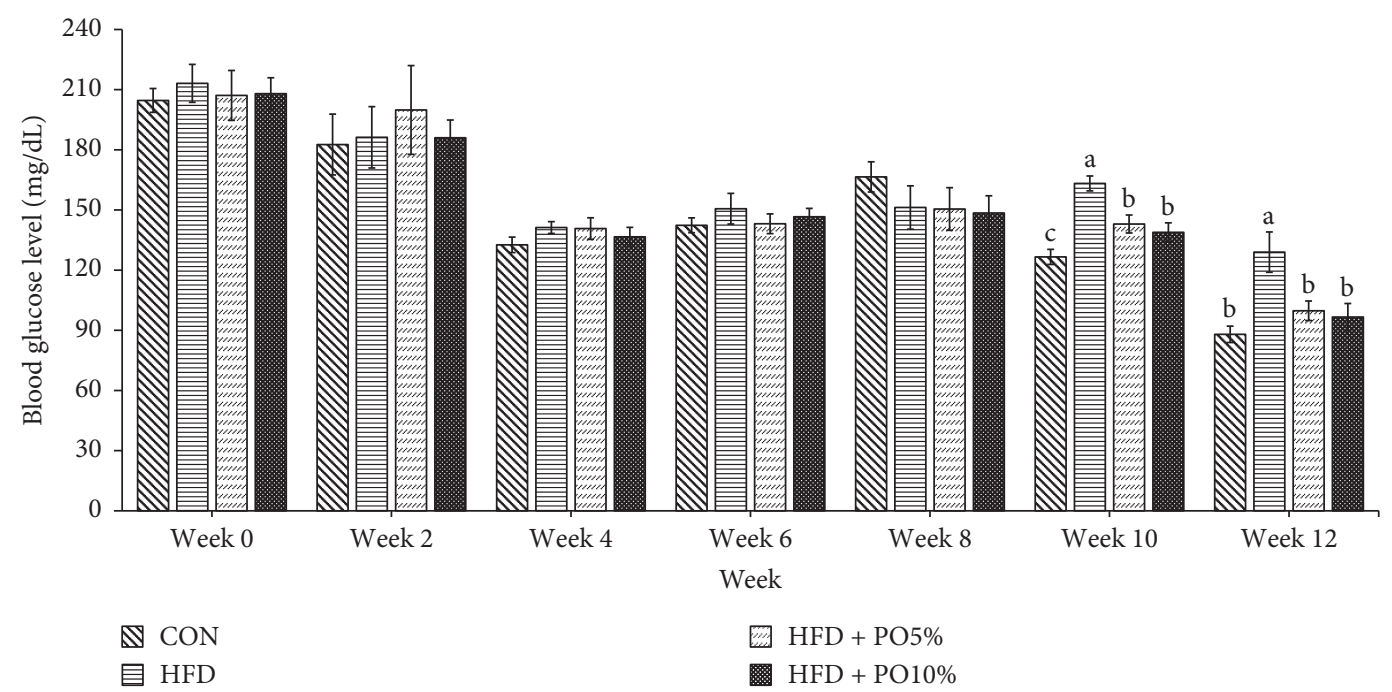

Figure 1: Effect of $P$. oleracea powder on random blood glucose levels at 0, 2, 4, 6, 8, 10, and 12 weeks in mice fed normal diet (CON), high fat diet (HFD), high fat diet with 5\% P. oleracea powder (HFD+PO5\%), and high fat diet with 10\% P. oleracea powder (HFD+PO10\%). Values are the mean $\pm \mathrm{SE}(n=8$ /group). The same letters represent no significant differences according to the Duncan's multiple range test. $p<0.05$ was considered significant.\$ CON: normal diet; 自 HFD: high fat diet; 图 HFD + PO5\%: high fat diet with 5\% P. oleracea powder; high fat diet with $10 \%$ P. oleracea powder.

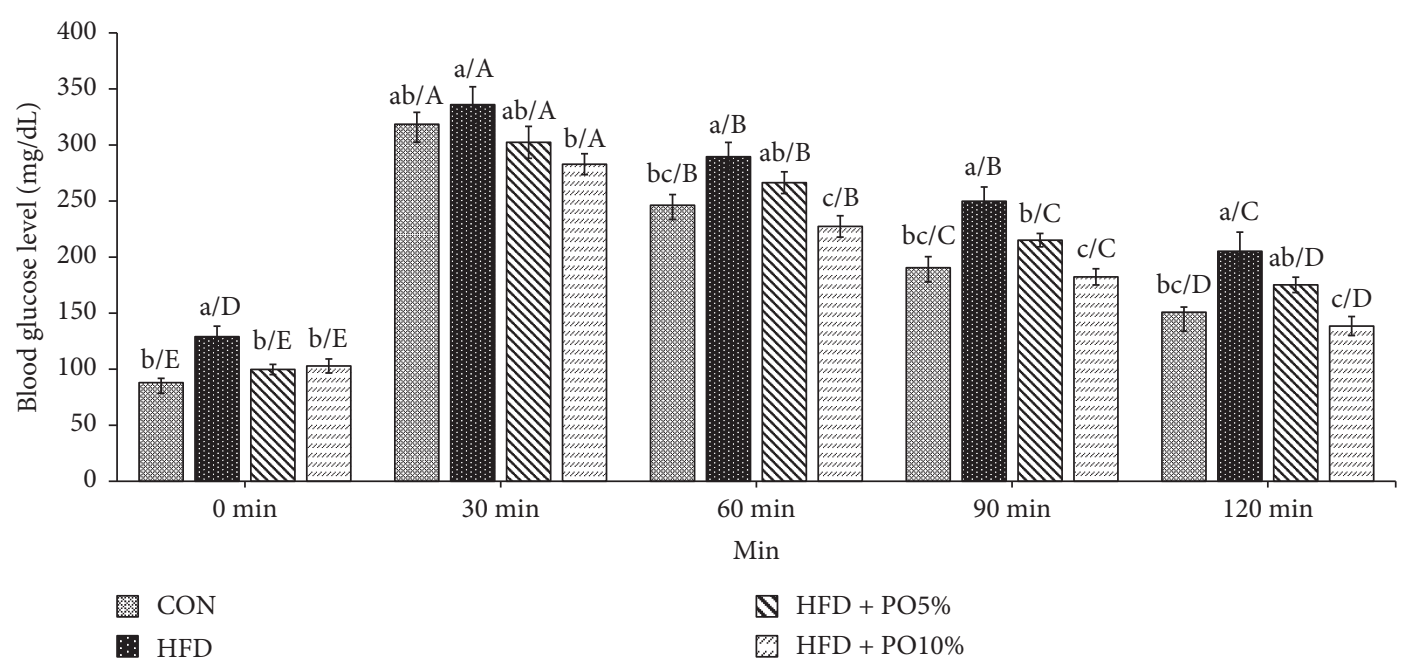

FIGURE 2: Effect of P. oleracea powder, normal diet, and high fat diet on IPGTT in C57BL/6 mice. Values are the mean \pm SE ( $n=8 /$ group). Different lowercase letters show significant differences between groups by Duncan's multiple range test $(p<0.05)$. Different capital letters

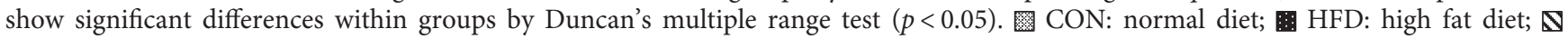

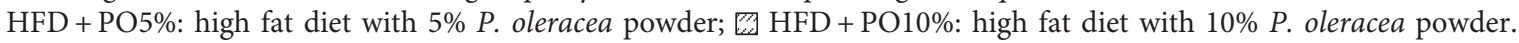

moderate centrilobular ballooning degeneration of the hepatocytes was observed in the HFD, HFD + PO5\%, and HFD + PO10\% groups (88\%, 25\%, and 25\%, respectively). These results suggest that the administration of $P$. oleracea powder suppressed liver lipid accumulation and hepatocyte cellular swelling.

3.7. Effects of P. oleracea Powder on the Expression of TNF- $\alpha$ Related to Inflammation. The protein expression level of TNF- $\alpha$ in liver tissue was measured via Western blot (Figures 4(a) and 4(b)). There were no observable differences between the CON and HFD groups, whereas the HFD + PO5\% and $\mathrm{HFD}+\mathrm{PO} 10 \%$ groups displayed decreased protein expression levels of TNF- $\alpha$ compared with those in the CON group $(p<0.05)$. When comparing the HFD groups only, the expression levels in the HFD + PO5\% and HFD + PO10\% groups were significantly lower than those in the HFD group.

3.8. Effects of P. oleracea Powder on Fatty Acid Oxidation in the Liver. The expression of PPAR- $\alpha$, a protein that controls fatty acid oxidation, was measured in liver tissue (Figures 4(a) and $4(c))$. No between-group differences were observed among 


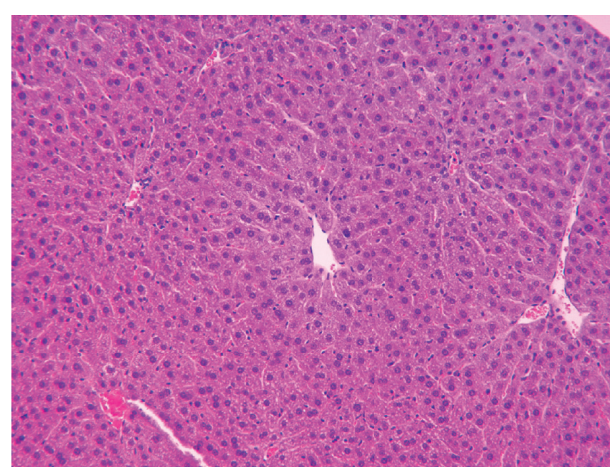

(a)

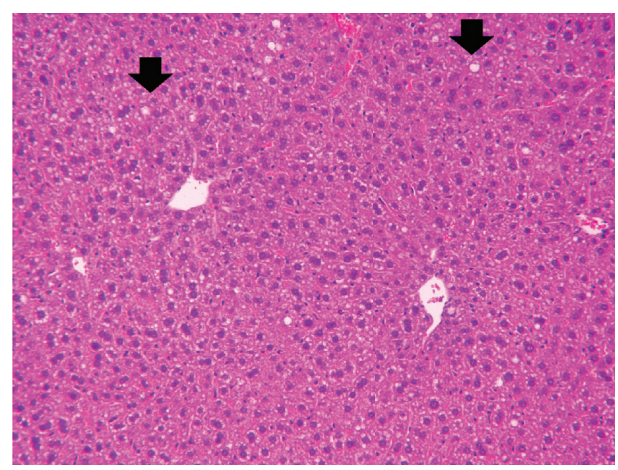

(c)

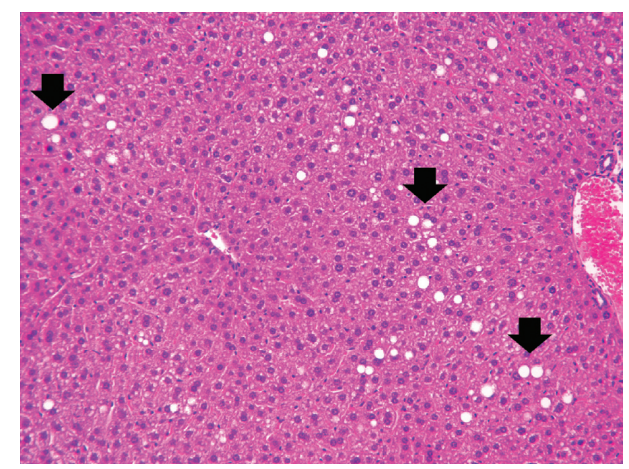

(b)

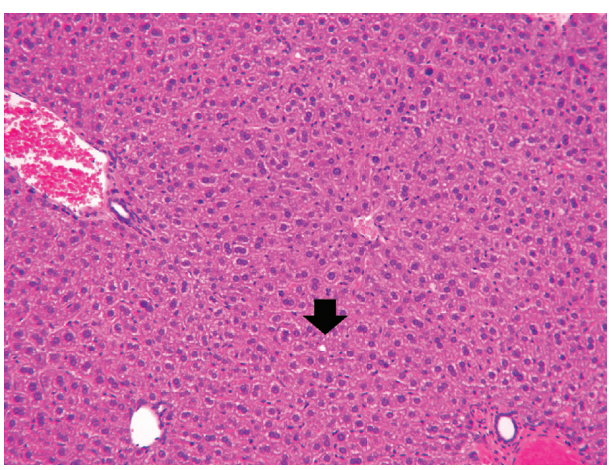

(d)

Figure 3: Histopathological analysis of liver in mice administered with high fat diet with low- or high-dose P. oleracea powder (5\% and 10\%, respectively). Arrows indicate the fatty degeneration of hepatocytes. (a) Negative control, (b) high fat diet (HFD), (c) high fat diet with 5\% P. oleracea powder (HFD+PO5\%), and (d) high fat diet with 10\% P. oleracea powder (HFD+PO10\%) (hematoxylin and eosin stain, x200 magnification).

the $\mathrm{CON}, \mathrm{HFD}+\mathrm{PO} 5 \%$, and HFD groups; however, the expression levels of PPAR- $\alpha$ in the HFD + PO10\% group were higher than those in the HFD group $(p<0.05)$.

3.9. Effects of P. oleracea Powder on Insulin Resistance in the Liver. The protein expression levels of GLUT4 and PPAR$\gamma$ were analyzed as indicators of insulin resistance (Figures 4(a), 4(d), and 4(e)). The GLUT4 expression level found in the HFD + PO10\% group was significantly higher than those in the CON, HFD, and HFD + PO5\% groups; however, no significant difference was observed among the latter three groups. Likewise, the protein expression level of PPAR- $\gamma$ was also found to be significantly higher in the HFD + PO10\% group than in the CON, HFD, and HFD + PO5\% groups; furthermore, there were no significant differences among the CON, HFD, and HFD + PO5\% groups.

\section{Discussion}

The diet-induced obese mice used in this study constitute an important model to elucidate the correlation between several chronic diseases and fat [22]. In a previous study, an HFD group fed a $P$. oleracea ethanol (PE) extract mix exhibited significantly decreased body weights compared with an HFD-fed group that did not receive PE [17]. In addition, Won and Kim [18] reported that the FER was significantly lower in mice treated with high-dose ethanol extracts from $P$. oleracea than in the HFD group. These observations were consistent with our results; they demonstrate that $P$. oleracea powder reduces body weight by decreasing FER and suppressing body weight gain. Although there were differences in diet calories by adding $P$. oleracea powder (5\% and $10 \%)$ in the HFD diet proportionally, the result of energy intake (kcal/day) showed no differences among the HFD groups $(p<0.05)$. That is, the overall efficacy of $P$. oleracea powder was due to $P$. oleracea powder itself rather than lesser diet calories.

In another study, epididymal fat was significantly reduced in stevia-treated groups compared with an HFD control (HC) group [23]. Additionally, the epididymal fat weight was significantly reduced in the doenjang-supplemented HFD group compared with the HFD group [24]. These observations were consistent with our results. PPAR- $\alpha$ is abundantly present in brown fat and the liver and is known to inhibit the growth and differentiation of adipocytes, promote lipolysis, and regulate lipoprotein synthesis and tissue inflammatory responses. This protein is also known to stimulate carnitine palmitoyltransferase I (CPT-1) to promote the $\beta$-oxidation of FFAs [8]. In this study, the HFD + PO10\% group exhibited the highest PPAR- $\alpha$ protein expression level. Administration of $P$. oleracea powder is 


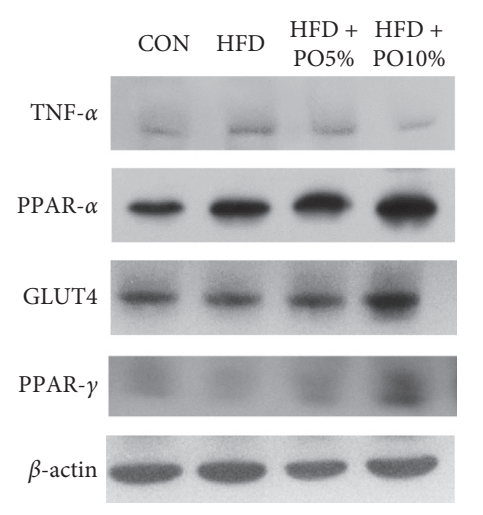

(a)

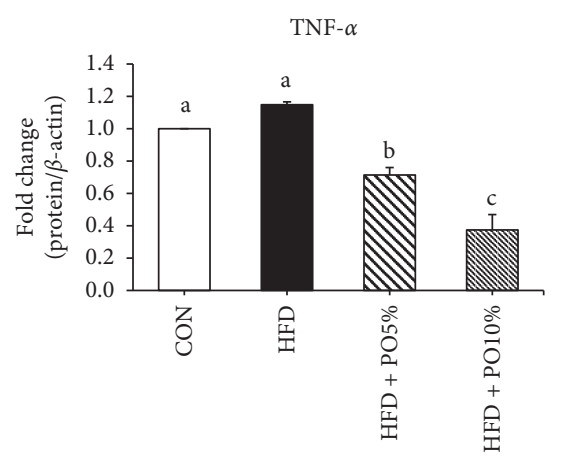

(b)

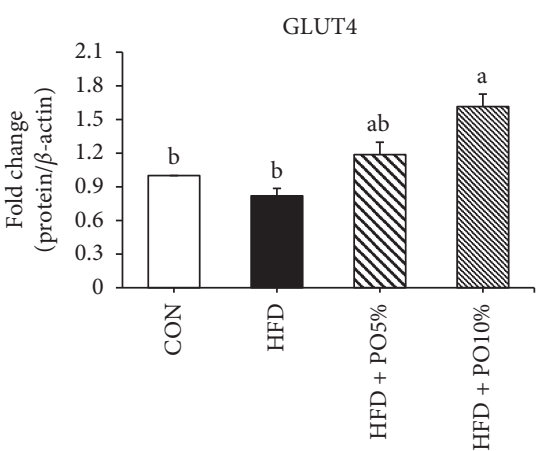

(d)

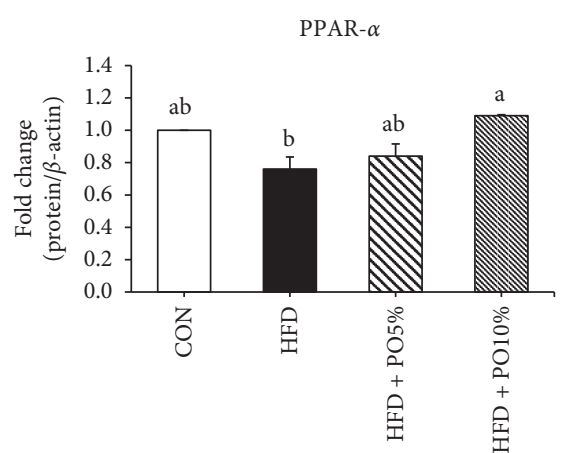

(c)

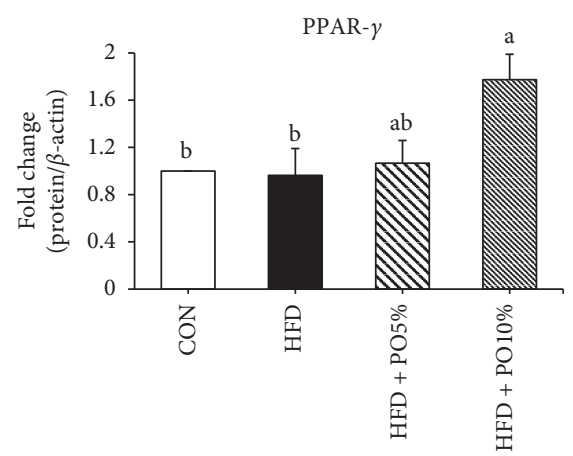

(e)

Figure 4: Effect of $P$. oleracea powder on relative protein expression in liver of C57BL/6 mice. Values are the mean \pm SE ( $n=8 /$ group). Values with different letters are significantly different at $p<0.05$ by Duncan's multiple range test. CON, normal diet; HFD, high fat diet; HFD+PO5\%, high fat diet with 5\% P. oleracea powder; HFD+PO10\%, high fat diet with $10 \%$ P. oleracea powder. (a) Representative western blot bands of TNF- $\alpha$, PPAR- $\alpha$, GLUT4, PPAR- $\gamma$, and $\beta$-actin. (b) Relative expression of TNF- $\alpha / \beta$-actin. (c) Relative expression of PPAR$\alpha / \beta$-actin. (d) Relative expression of GLUT4/ $\beta$-actin. (e) Relative expression of PPAR- $\gamma / \beta$-actin.

thought to inhibit fat accumulation by promoting fatty acid oxidation in the body through increased PPAR- $\alpha$ expression.

However, brown fat, which is characterized by energy consumption and heat generation, is known to play an important role in adaptive thermogenesis to allow for the maintenance of heat balance and basic body temperature $[25,26]$. This role of brown fat is known to be regulated by uncoupling proteins (UCPs) present in the inner mitochondrial membrane [26]. Previous studies have reported that the weight of brown fat per body weight unit is significantly higher in HFD groups than in normal chow groups and treated groups $[27,28]$. Although the fat pad weight of brown adipose tissue in the royal jelly (RJ) group was lower, it was reported that the RJ group had higher levels of UCP-1 and cytochrome $\mathrm{c}$ oxidase subunit IV (COX-IV) protein expression, thereby increasing thermogenesis for energy consumption [28]. In this study, the brown fat weight in the HFD groups was also higher than that of the CON group. There was no difference in the weight of brown fat between the HFD + PO10\% and HFD groups; however, based on previous studies' results, body weight is likely controlled through thermogenesis for energy consumption via the expression of both UCP-1 and COX-IV. Additional studies are required to confirm this hypothesis.

Obesity reportedly increases blood levels of TC, LDLcholesterol, VLDL-cholesterol, and TG while also decreasing
HDL-cholesterol levels. This increases the likelihood that an obese subject will experience cardiovascular diseases such as hyperlipidemia, hypercholesterolemia, and atherosclerosis $[2,29]$. Additionally, chronically excessive fat intake is known to affect vascular health through oxidative and inflammatory reactions caused by metabolic stress [30]. In a previous study, HDL-cholesterol levels were higher in garlic shoot extract-treated groups than in a control group, whereas LDL-cholesterol and VLDL-cholesterol levels were lower, and the AI and CRF were significantly lower [31]. The $\mathrm{AI}$ is known as a risk indicator for coronary artery disease and CRF is known as a hyperlipidemia risk indicator [32]. In our study, the HDL-cholesterol, AI, and CRF values in the $\mathrm{HFD}+\mathrm{PO} 5 \%$ and the HFD + PO10\% groups were significantly lower than those in the HFD group; therefore, administration of $P$. oleracea powder may be an effective measure to prevent cardiovascular disease.

Furthermore, TNF- $\alpha$, a proinflammatory cytokine, is known to regulate leukocyte adhesion and migration in vascular inflammatory diseases including atherosclerosis [33]. In our study, $P$. oleracea powder administration resulted in significantly lower TNF- $\alpha$ protein expression in the $P$. oleracea-treated groups than in the HFD group (Figures 4(a) and 4(b)). According to a study, an aqueous extract of $P$. oleracea reportedly prevented vascular 
inflammatory processes not only by inhibiting intracellular reactive oxygen species (ROS) production and nuclear factor $(\mathrm{NF})-\kappa \mathrm{B}$ activation but also by reducing the overexpression of vascular cell adhesion molecule 1 (VCAM-1), intercellular adhesion molecule 1 (ICAM-1), and endothelial cell selectin (E-selectin) [34]. Therefore, the administration of $P$. oleracea powder both improves blood lipid fractions and cardiovascular diseases such as atherosclerosis.

Additionally, an HFD is known to induce hyperinsulinemia and hyperglycemia [3], and increased serum insulin levels and HOMA-IR values are indicative of insulin resistance [35]. In this study, blood glucose levels at week 10 and week 12 were lower in the $P$. oleracea-treated group than in the HFD group $(p<0.05)$. The HOMA-IR and IPGTT values were lower in the HFD $+\mathrm{PO} 10 \%$ group than in the HFD group $(p<0.05)$.

A previous study involved 4-week-old C57BL/6 mice being orally administered with $0 \%, 0.2 \%, 0.5 \%$, and $1 \%$, respectively, of Artemisia Princeps ethanol (APE) extract once a day for 14 weeks [36]. In the normal diet and high-fat diet, the blood glucose level in the oral glucose tolerance test (OGTT) was lower in the HF-1.0\%APE group than in the HF-0\%APE group $(p<0.05)$, plasma insulin level was not a significant difference between the HF-0.5\%APE group and HF-0\%APE group, but the HF-0.5\%APE group was lower than the HF-0\% APE group in HOMA-IR $(p<0.05)$ [37]. These results show that glucose tolerance can be improved by improving the HOMA-IR value, which is a good predictor of insulin sensitivity [37]. In a previous study, an HFD group exhibited the highest HOMA-IR value $(p<0.05)$, whereas the values in mulberry anthocyanin groups were significantly higher than those in a low-fat diet (LFD) group [35]. These observations are consistent with our results. Therefore, $P$. oleracea may reduce HOMA-IR values, which in turn relieves type 2 diabetes by decreasing insulin resistance.

Adiponectin is an adipokine that is exclusively secreted from adipose tissue into the bloodstream and accounts for $0.01 \%$ of total plasma protein. Oral administration of adiponectin to obese and diabetic mice results in decreases in both body weight and blood glucose levels and enhanced insulin sensitivity [9]. TNF- $\alpha$ secretion increases dramatically in both obese subjects and patients with diabetes, and it directly contributes to the reduction of adiponectin [10]. Sahin et al. [10] demonstrated that the expression levels of PPAR $-\gamma$ in treated groups were significantly higher than those in an HFD/streptozotocin (STZ) group. Additionally, the expression of PPAR- $\gamma$ and adiponectin was higher in the treated group than in the HFD group $(p<0.05)$, whereas the expression of TNF- $\alpha$ was significantly lower [23]. Our results in this study were consistent with those of the abovementioned study.

An increase in GLUT4 expression is known to reduce insulin resistance [38]. Glucose utilization decreases in insulin-resistant muscle, and the expression of GLUT4 protein and mRNA in skeletal muscle decreases with an HFD [37]. In previous studies, GLUT4 and PPAR- $\gamma$ protein expressions were significantly increased in treated groups compared with a diabetic control group [39, 40]; these observations were consistent with our results.

\section{Conclusion}

In conclusion, our findings indicate that body weight gain and perirenal and epididymal fat tissues were reduced significantly in the high $P$. oleracea powder group along with increased HDL-cholesterol and decreased HOMA-IR. PPAR- $\alpha$, GLUT4, and PPAR- $\gamma$ protein expression were upregulated in the high $P$. oleracea powder group compared to the HFD group, while TNF- $\alpha$ protein expression was downregulated in the $P$. oleracea-treated groups than in the HFD group. We concluded that the $P$. oleracea powder was effective at suppressing body weight gain, reducing body fat, regulating blood glucose level and insulin sensitivity, and decreasing inflammation.

\section{Data Availability}

The data used to support the findings of this study are available from the corresponding author upon request.

\section{Conflicts of Interest}

The authors declare that they have no conflicts of interest.

\section{Authors' Contributions}

All the authors contributed to the study conception and design. Material preparation, data collection, and analysis were performed by Jae Hyun Jung, Su Bin Hwang, Hyeon Ju Park, and Guang-Ri Jin. The first draft of the manuscript was written by Jae Hyun Jung. Bog Hieu Lee made final editing and proofreading of the revised manuscript. All the authors read and approved the final manuscript.

\section{Acknowledgments}

This work was supported by the Basic Science Research Program through the National Research Foundation of Korea (NRF) funded by the Ministry of Education, Science and Technology (NRF-2017R1D1A1B03033507).

\section{References}

[1] E. Riant, A. Waget, H. Cogo, J.-F. Arnal, R. Burcelin, and P. Gourdy, "Estrogens protect against high-fat diet-induced insulin resistance and glucose intolerance in mice," Endocrinology, vol. 150, no. 5, pp. 2109-2117, 2009.

[2] G. H. Tomkin, "Atherosclerosis, diabetes and lipoproteins," Expert Review of Cardiovascular Therapy, vol. 8, no. 7, pp. 1015-1029, 2010.

[3] R. Buettner, J. Schölmerich, and L. C. Bollheimer, "High-fat diets: modeling the metabolic disorders of human obesity in rodents," Obesity, vol. 15, no. 4, pp. 798-808, 2007.

[4] J. Y. Lee, Y.-R. Lee, H.-R. Kim, J.-P. Myong, and M.-Y. Kang, "Trends in obesity prevalence by occupation based on Korean national health and nutrition examination survey from 1998 to 2015," Safety and Health at Work, vol. 11, no. 1, pp. 97-102, 2020.

[5] M. Jeszka-Skowron, E. Flaczyk, J. Jeszka, Z. Krejpcio, E. Król, and M. S. Buchowski, "Mulberry leaf extract intake reduces hyperglycaemia in streptozotocin (STZ)-induced diabetic rats 
fed high-fat diet," Journal of Functional Foods, vol. 8, pp. 9-17, 2014.

[6] I. Baik, "Projection of diabetes prevalence in Korean adults for the year 2030 using risk factors identified from national data," Diabetes \& Metabolism Journal, vol. 43, no. 1, pp. 90-93, 2019.

[7] I.-S. Ahn, K.-Y. Park, and M.-S. Do, "Weight control mechanisms and antiobesity functional agents," Journal of the Korean Society of Food Science and Nutrition, vol. 36, no. 4, pp. 503-513, 2007.

[8] J. H. Shon and T. Y. Kim, "Recent update on pathogenesis of nonalcoholic fatty liver disease," Korean Journal of International Medicine, vol. 79, no. 5, pp. 461-474, 2010.

[9] C. Tian, X. Ye, R. Zhang et al., "Green tea polyphenols reduced fat deposits in high fat fed rats via erk1/2-PPAR- $\gamma$-adiponectin pathway," PLoS One, vol. 8, no. 1, pp. 1-9, 2013.

[10] K. Sahin, M. Tuzcu, C. Orhan et al., "Antidiabetic activity of chromium picolinate and biotin in rats with type 2 diabetes induced by high-fat diet and streptozotocin," British Journal of Nutrition, vol. 110, no. 2, pp. 197-205, 2013.

[11] J. H. Park, S. H. Lee, I.-M. Chung, and Y. Park, "Sorghum extract exerts an antidiabetic effect by improving insulin sensitivity via PPAR- $\gamma$ in mice fed a high-fat diet," Nutrition Research and Practice, vol. 6, no. 4, pp. 322-327, 2012.

[12] Y.-X. Zhou, H.-L. Xin, K. Rahman, S.-J. Wang, C. Peng, and H. Zhang, "Portulaca oleracea L.: a review of phytochemistry and pharmacological effects," BioMed Research International, vol. 2015, Article ID 925631, 11 pages, 2015.

[13] M.-J. Kim, S.-J. Lee, R.-J. Kim, B.-Y. Jeong, and N.-J. Sung, "Mineral content and antioxidants activity of Portulaca oleracea," Journal of Life Science, vol. 21, no. 10, pp. 1393-1400, 2011.

[14] S. Y. Hong and S. N. Kim, "Scaling of the scalp with alcohol extract of Portulaca oleracea," Korean Journal of Aesthet Cosmetology, vol. 10, no. 3, pp. 725-729, 2012.

[15] J. H. Lee, J. E. Park, and J. S. Han, "Portulaca oleracea L. extract reduces hyperglycemia via PI3k/Akt and AMPK pathways in the skeletal muscles of C57BL/Ksj-db/db mice," Journal of Ethnopharmacology, vol. 260, Article ID 112973, 2020.

[16] B. Nemzer, F. Al-Taher, and N. Abshiru, "Phytochemical composition and nutritional value of different plant parts in two cultivated and wild P. oleracea (Portulaca oleracea L.) genotypes," Food Chemistry, vol. 320, Article ID 126621, 2020.

[17] K.-S. Kang, "Effects of purslane extract on obesity and diabetes in high-fat diet-induced obese mice," Journal of the Korea Society of Computer and Information, vol. 21, no. 7, pp. 61-66, 2016.

[18] H.-R. Won and S.-H. Kim, "Antihyperlipidemic effect of diet containing Portulaca oleracea L. ethanol extract in high fat diet-induced obese mice," Journal of the Korean Society of Food Science and Nutrition, vol. 40, no. 4, pp. 538-543, 2011.

[19] D. Gao, Q. Li, and Y. Fan, "Hypoglycemic effects and mechanisms of Portulaca oleracea L. in alloxan-induced diabetic rats," Journal of Medicinal Plants Research, vol. 4, no. 19, pp. 1996-2003, 2010.

[20] T.-H. Kim, Y.-K. Son, K.-H. Hwang, and M.-H. Kim, "Effects of angelica keiskei koidzumi and turmeric extract supplementation on serum lipid parameters in hypercholesterolemic diet or P-407-Induced hyperlipidemic rats," Journal of the Korean Society of Food Science and Nutrition, vol. 37, no. 6, pp. 708-713, 2008.

[21] K. K. Trout, C. Homko, and N. C. Tkacs, "Methods of measuring insulin sensitivity," Biological Research For Nursing, vol. 8, no. 4, pp. 305-318, 2007.
[22] C.-Y. Wang and J. K. Liao, "A mouse model of diet-induced obesity and insulin resistance," Methods in Molecular Biology, vol. 821, pp. 421-433, 2011.

[23] J. E. Park, H. J. Kee, and Y. S. Cha, "Effect of Stevia rebaudiana bertoni leaf extract on antiobesity in C57BL/6J mice," Korean Journal of Food Science and Technology, vol. 42, no. 5, pp. 586-592, 2010.

[24] C.-R. Bae, D. Y. Kwon, and Y.-S. Cha, "Anti-obesity effects of salted and unsalted doenjang supplementation in C57BL/6J mice fed with high fat diet," Journal of the Korean Society of Food Science and Nutrition, vol. 42, no. 7, pp. 1036-1042, 2013.

[25] S. Gesta, Y.-H. Tseng, and C. R. Kahn, "Developmental origin of fat: tracking obesity to its source," Cell, vol. 131, no. 2, pp. 242-256, 2007.

[26] G. Alberdi, V. M. Rodríguez, J. Miranda, M. T. Macarulla, I. Churruca, and M. P. Portillo, "Thermogenesis is involved in the body-fat lowering effects of resveratrol in rats," Food Chemistry, vol. 141, no. 2, pp. 1530-1535, 2013.

[27] C. Roberts-Toler, B. T. O’Neill, and A. M. Cypess, “Dietinduced obesity causes insulin resistance in mouse brown adipose tissue," Obesity, vol. 23, no. 9, pp. 1765-1770, 2015.

[28] T. Yoneshiro, R. Kaede, K. Nagaya et al., "Royal jelly ameliorates diet-induced obesity and glucose intolerance by promoting brown adipose tissue thermogenesis in mice," Obesity Research \& Clinical Practice, vol. 12, no. 1, pp. 127137, 2018.

[29] W. B. Kannel and P. W. F. Wilson, "An update on coronary risk factors," Medical Clinics of North America, vol. 79, no. 5, pp. 951-971, 1995.

[30] Y. N. Lim, O. R. Kwon, and J. Y. Kim, "The model for evaluation on blood flow of functional food in human intervention study," Journal of Lipid and Atherosclerosis, vol. 7, no. 2, pp. 88-97, 2018.

[31] C.-R. Hwang, J.-R. Kang, M.-J. Kang, H.-J. Sim, and J.-H. Shin, "Effects of garlic shoot extract on lipid metabolism in hyperlipidemic rats fed a high-fat diet," Journal of Life Science, vol. 25, no. 3, pp. 276-284, 2015.

[32] H. J. Kang, I. H. Jeon, T. O. Kwon et al., "Effect of Mori Folium extract on improvement of blood flow in ferric chloride-induced carotid artery damage rat model," Journal of Physiology \& Pathology Korean Medicine, vol. 28, no. 6, pp. 607-613, 2014.

[33] T. Minami and W. C. Aird, "Endothelial cell gene regulation," Trends in Cardiovascular Medicine, vol. 15, no. 5, pp. 174-184, 2005.

[34] A. S. Lee, J. S. Kim, Y. J. Lee, D. G. Kang, and H. S. Lee, “AntiTNF- $\alpha$ activity of Portulaca oleracea in vascular endothelial cells," International Journal of Molecular Sciences, vol. 13, no. 5, pp. 5628-5644, 2012.

[35] T. Wu, X. Qi, Y. Liu et al., "Dietary supplementation with purified mulberry (Morus australis poir) anthocyanins suppresses body weight gain in high-fat diet fed C57BL/6 mice," Food Chemistry, vol. 141, no. 1, pp. 482-487, 2013.

[36] N. Yamamoto, Y. Kanemoto, M. Ueda, K. Kawasaki, I. Fukuda, and H. Ashida, "Anti-obesity and antidiabetic effects of ethanol extract of Artemisia princeps in C57BL/6 mice fed a high-fat diet," Food Function, vol. 2, no. 1, pp. 45-52, 2011.

[37] H. Meada, M. Hosokawa, T. Sasima, K. Murakami-Funayama, and K. Miyashita, "Anti-obesity and antidiabetic effects of fucoxanthin on diet-induced obesity conditions in a murine model," Research in Molecular Medicine, vol. 2, no. 6, pp. 897-902, 2009. 
[38] N. Ikarashi, T. Toda, T. Okaniwa et al., "Anti-obesity and antidiabetic effects of acacia polyphenol in obese diabetic KKAy mice fed high-fat diet," Evidence Based Complementary and Alternative Medicine, vol. 2011, Article ID 952031, 10 pages, 2011.

[39] A. K. Singh, V. Raj, A. K. Keshari et al., "Isolated mangiferin and naringenin exert antidiabetic effect via PPAR $\gamma /$ GLUT4 dual agonistic action with strong metabolic regulation," Chemico-Biological Interactions, vol. 280, pp. 33-44, 2018.

[40] C. Sunil, V. Duraipandiyan, P. Agastian, and S. Ignacimuthu, "Antidiabetic effect of plumbagin isolated from Plumbago zeylanica L. root and its effect on GLUT4 translocation in streptozotocin-induced diabetic rats," Food and Chemical Toxicology, vol. 50, no. 12, pp. 4356-4363, 2012. 